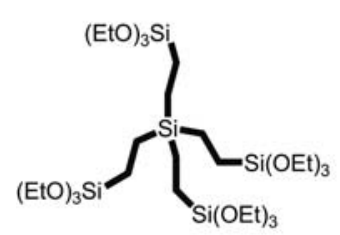

1

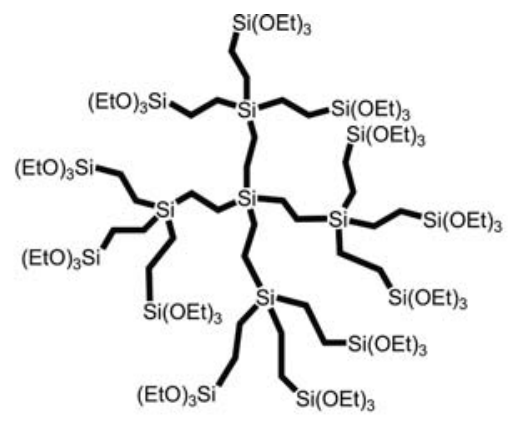

2

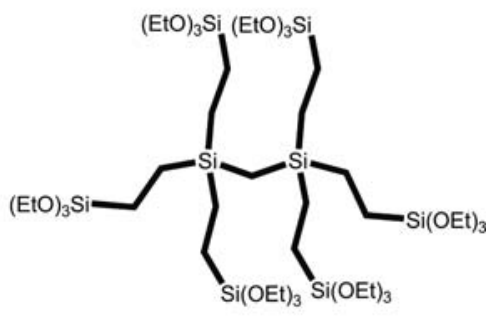

3

Scheme 1.

$\mathrm{SiC}_{4}$ units. The integral ratios were reproducible, with varying pulse delay times between 3 and $30 \mathrm{~s}$, indicating that the ${ }^{29} \mathrm{Si}$ nuclei were sufficiently relaxed to give accurate integrals. Nitrogen isotherms demonstrated the ordered mesoporous nature of both PMD-2 and PMD3 by showing typical type-IV isotherms. The BET surface areas were $775 \mathrm{~m}^{2} \mathrm{~g}^{-1}$ (PMD-2) and $767 \mathrm{~m}^{2} \mathrm{~g}^{-1}$ (PMD-3). The BJH analysis revealed a narrow pore size distribution with average pore sizes of $9.1 \mathrm{~nm}$ (PMD-2) and 8.2 nm (PMD-3), which is consistent with the TEM data.
References and Notes

1. I. W. Hamley, Angew. Chem. Int. Ed. Engl. 42, 1692 (2003).

2. G. J. de A. A. Soler-Illia, C. Sanchez, B. Lebeau, J. Patarin, Chem. Rev. 102, 4093 (2002).

3. G. R. Newkome, E. He, C. N. Moorefield, Chem. Rev. 99, 1689 (1999).

4. M. Fischer, F. Vögtle, Angew. Chem. Int. Ed. Engl. 38, 884 (1999).

5. C. T. Kresge, M. Leonowicz, J. C. Vartuli, J. S. Beck, Nature 359, 710 (1992).

6. A. W. Van der Made, P. W. N. M. Van Leeuwen, Chem. Commun. 1400 (1992).

7. M. J. Michalczyk, W. J. Simonsick Jr., K. G. Sharp, J. Organomet. Chem. 521, 261 (1996).

8. D. Seyferth, D. Y. Son, A. L. Rheingold, R. L. Ostrander, Organometallics 13, 2682 (1994).

\title{
A Reversible Synthetic Rotary Molecular Motor
}

\author{
José V. Hernández, Euan R. Kay, David A. Leigh*
}

\begin{abstract}
The circumrotation of a submolecular fragment in either direction in a synthetic molecular structure is described. The movement of a small ring around a larger one occurs through positional displacements arising from biased Brownian motion that are kinetically captured and then directionally released. The sense of rotation is governed solely by the order in which a series of orthogonal chemical transformations is performed. The minimalist nature of the [2] catenane flashing ratchet design permits certain mechanistic comparisons with the Smoluchowski-Feynman ratchet and pawl. Even when no work has to be done against an opposing force and no net energy is used to power the motion, a finite conversion of energy is intrinsically required for the molecular motor to undergo directional rotation. Nondirectional rotation has no such requirement.
\end{abstract}

Molecular-level motors differ from their macroscopic counterparts not only in scale but in how environmental factors influence their operation. Macroscopic machines are generally unaffected by ambient thermal

School of Chemistry, University of Edinburgh, The King's Buildings, West Mains Road, Edinburgh EH9 3JJ, UK.

*To whom correspondence should be addressed. E-mail: David.Leigh@ed.ac.uk energy, and a directional force must be applied to cause movement of each component. For molecular-sized motors, however, inertia is negligible and the parts are subject to random and incessant Brownian motion (1). Rather than fight this effect, biological motors use these random fluctuations in their mechanisms (2). For example, in $\mathrm{F}_{1} \mathrm{~F}_{0}-$ adenosine triphosphatase (ATPase), which spins the $\gamma$ shaft counterclockwise (viewing $\mathrm{F}_{1}$ from above) as proton-motive force
9. Materials and methods are available as supporting material on Science Online.

10. S. Inagaki, S. Guan, Y. Fukushima, T. Ohsuna, O. Terasaki, J. Am. Chem. Soc. 121, 9611 (1999).

11. The authors thank Dr. N. Coombs for the TEM imaging. G.A.O. is Government of Canada Research Chair in Materials Chemistry. $\mathrm{He}$ is indebted to the Natural Sciences and Engineering Research Council of Canada for support of this work.

Supporting Online Material www.sciencemag.org/cgi/content/full/306/5701/1529/ DC1

Materials and Methods

26 August 2004; accepted 13 October 2004

powers ATP production and clockwise if ATP is consumed to drive proton flow against a concentration gradient (3), Brownian motion drives both the power and exhaust strokes (2). Inspired by such biological motors and by Feynman's celebrated discussion (4) of the miniature ratchet and pawl first introduced (5) by Smoluchowski, efforts have been made to design molecules that exhibit directional control over submolecular rotary motion (6-10). Unidirectional rotation about single $(11,12)$, double $(13-16)$, and mechanical (17) bonds has been achieved, but unlike $\mathrm{F}_{1} \mathrm{~F}_{0}$-ATPase, these artificial motor molecules are only able to rotate in one direction but not the other. We now report on a molecular structure in which a fragment can be circumrotated in either direction, and we probe features of the underlying mechanism.

During the past decade, a number of remarkable theoretical formalisms have been developed using nonequilibrium statistical physics that explain how various types of fluctuation-driven transport can occur $(18,19)$. Underlying each of these Brownian ratchet or motor mechanisms are three components (20): (i) a randomizing element (21), (ii) an energy input to avoid falling foul of the Second Law of Thermodynamics $(22,23)$, and (iii) asymmetry in the energy or information potential in the dimension in which the motion occurs. 
Fig. 1. A flashing energy ratchet mechanism for Brownian particle transport along an oscillating potential energy surface.

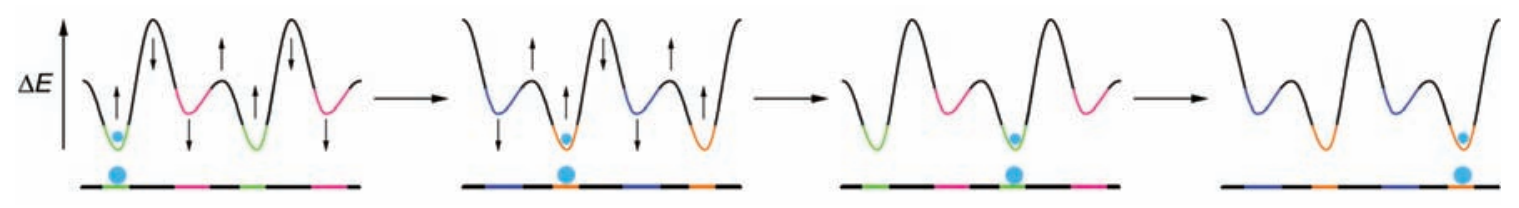

Such ratchet mechanisms not only account for the general principles behind biological motors (24-27) but have also been successfully applied to the development of transport and separation devices for mesoscopic particles and macromolecules, microfluidic pumping, the photoalignment of liquid crystals, and quantum and electronic applications $(26,28-31)$.

A flashing ratchet is a particular type of energy ratchet mechanism (25), a classic example of which consists in physical terms (Fig. 1) of an asymmetric potential energy surface (a periodic series of two different minima and two different maxima) along which a Brownian particle is directionally transported by sequentially raising and lowering each set of minima and maxima by changing the potential (for example, with an oscillating electric field and a charged particle). Catenanes and rotaxanes, molecules in which components are physically linked together but not connected by covalent bonds, are excellent systems with which to study characteristics of submolecular motion (8). The mechanical linkage inherently restricts certain degrees of freedom for the relative displacement of the interlocked components while simultaneously permitting large-amplitude motion in the allowed vectors. The way in which the principles of an energy ratchet can be applied to a catenane architecture is not to consider the whole structure as a molecular machine, but rather to view one macrocycle as a motor that transports a substrate- the other ring - directionally around itself. In its simplest manifestation, this gives rise to a [2]catenane such as $\mathbf{1}$ (Fig. 2) that should be able to unidirectionally rotate the smaller ring about the larger one in response to a series of chemical reactions.

There are several differences, however, between the flashing ratchet particle transport mechanism in Fig. 1 and the one applied to catenane $\mathbf{1}$ in Fig. 2: (i) The molecular system is cyclic, so the translational transport along a periodic energy potential becomes a directional rotation around a two-minima-two-maxima loop; (ii) only one energy minimum is varied for 1, not both (which is sufficient to ensure that the energy difference between the two minima changes twice); and (iii) the single steps that simultaneously change minima depth and maxima height in the classical energy ratchet mechanism are separated into their thermodynamic and kinetic con-
Fig. 2. (A) Schematic illustration and (B) potential energy surface for the small light blue ring in a minimalist [2]catenane rotary molecular motor, 1. The ring preferentially resides on one or other of the two binding sites (stations), represented by colored cylinders. The colored spheres are bulky groups, each of which sterically blocks one of the two tracks between the stations. The blue-to-green and green-to-blue transformations represent (balance-breaking) chemical reactions that change the binding affinity of a station for the small ring, providing a driving force for the ring to redistribute itself between the stations if it is able to be exchanged between them.
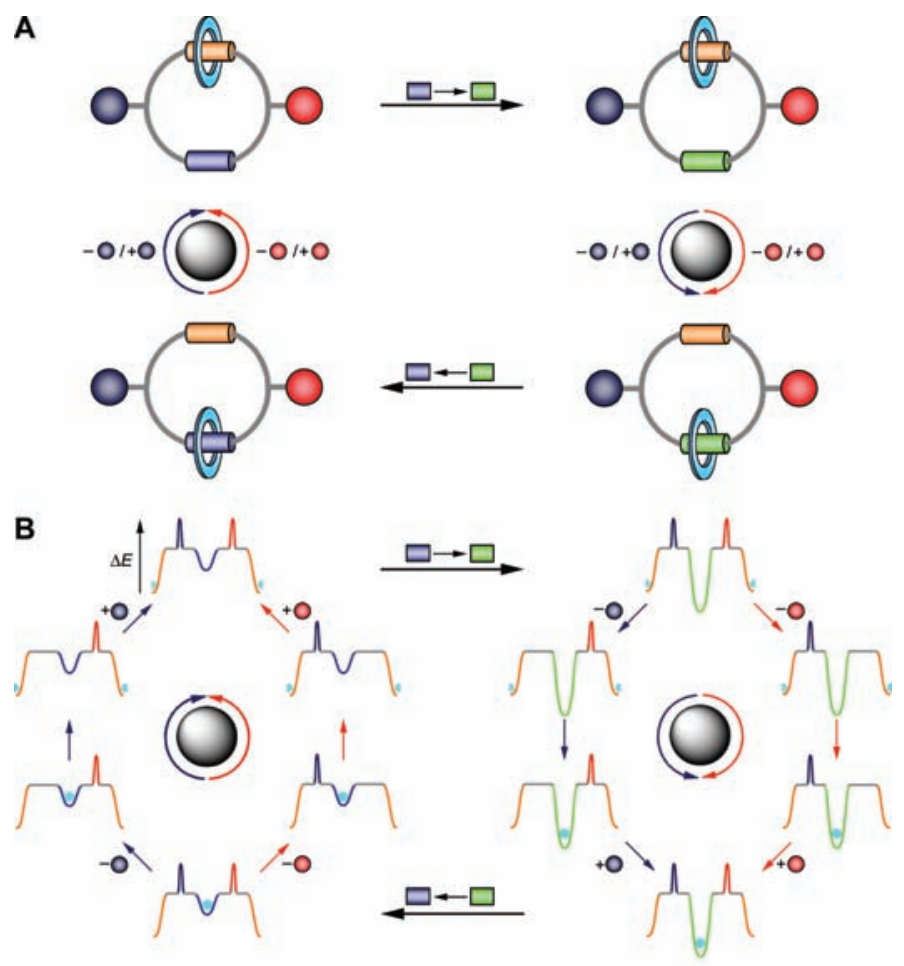

Station affinity: $\square>\square>\square$

purple sphere (linking reaction) allows the ring to move between stations by a particular route. Reattachment of the sphere (unlinking reaction) ratchets the net transported quantity of rings.

stituents in the chemical system. Despite these differences, the physical principles behind the two mechanisms are the same.

In chemical terms, catenane $\mathbf{1}$ is a stimuli-responsive molecular shuttle $(8,32)$ with two routes (each of which can be independently blocked) that connect the two binding sites or "stations" for the small macrocycle (Fig. 3). To satisfy oneself that internal motion within a chemical structure can be described in terms of a stochastic transport mechanism, it is useful to consider how the net change of position occurs within a typical stimuli-responsive molecular shuttle (32). At equilibrium, the small macrocycle is distributed between two different stations (an asymmetric track) according to a Boltzmann distribution. An external trigger (the energy input) chemically modifies one binding site in such a way that the initially disfavored station becomes energetically more favorable for the macrocycle. Thermal fluctuations (the randomizing element) provide the energy required by the small macrocycle to sever its interactions with a station and set off on a Brownian walk through which the new equilibrium position is established.

Catenane 1 was prepared as the fum- $E-1$ (33) isomer according to Fig. $3(34,35)$. Net changes in the position or potential energy of the smaller ring were sequentially achieved by (i) photoisomerization to the maleamide $(\rightarrow$ mal-Z-1) $\quad(36,37)$; (ii) de-silylation/resilylation $(\rightarrow$ succ-Z-1); (iii) reisomerization to the fumaramide $(\rightarrow$ succ-E-1); and finally, (iv) de-tritylation/re-tritylation to regenerate fum- $E-\mathbf{1}$, the whole reaction sequence producing a net clockwise (as drawn in Fig. 3) circumrotation of the small ring about the larger one. Exchanging the order of steps (ii) and (iv) generated the equivalent counterclockwise rotation of the small ring. The time scales and number of reactions involved for directional rotation in $\mathbf{1}$ make it somewhat less practical than the methods previously developed for nonreversible synthetic rotary motors $(11-17)$. Nevertheless, the chemistry is surprisingly robust and can be carried out as a direct sequence of reactions without purification at each stage, using resins to neutralize or remove excess re- 

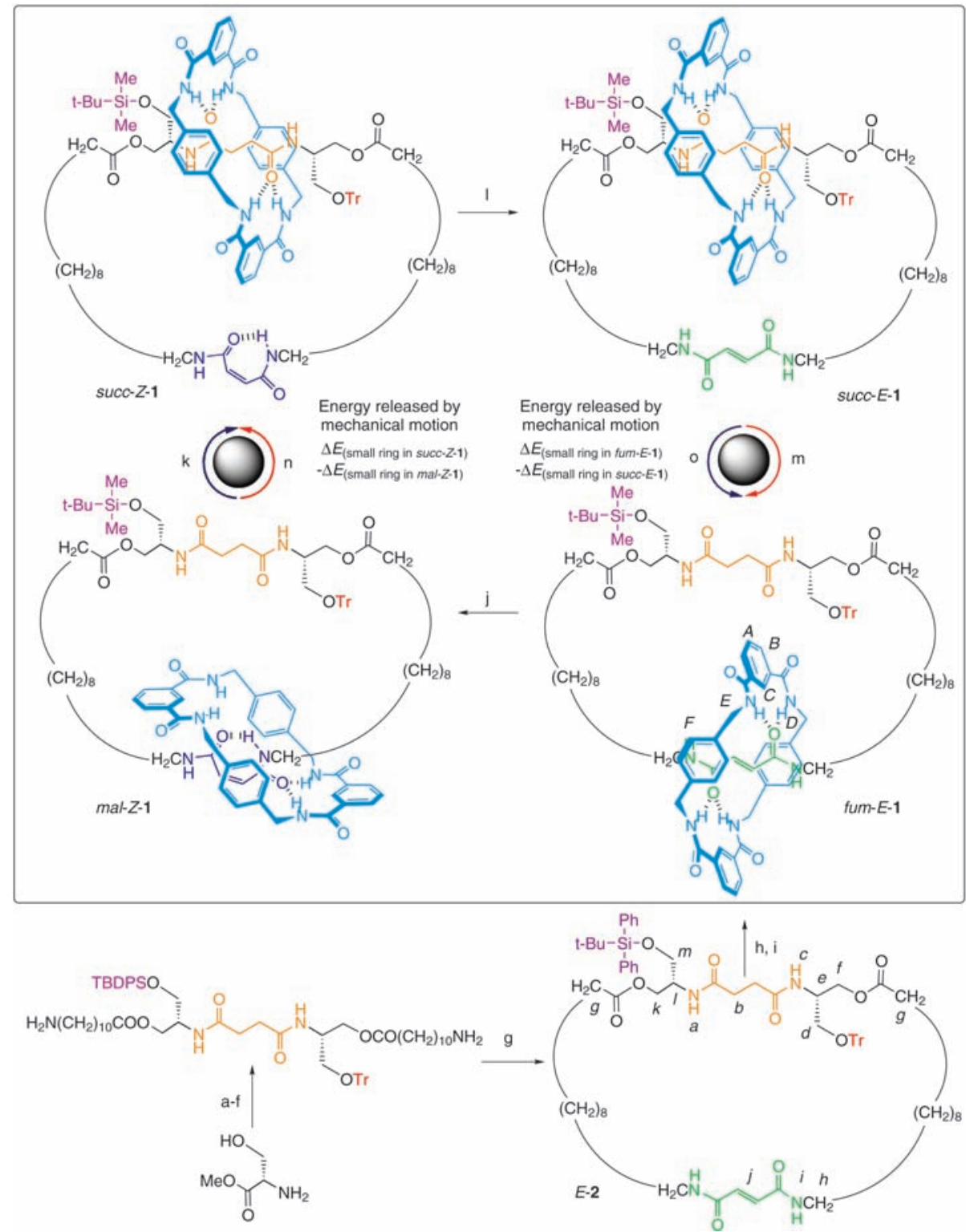

Fig. 3. Synthesis route and operation of reversible rotary motor 1 . Reagents and conditions (unless otherwise stated, reactions were carried out at room temperature and in $\mathrm{CH}_{2} \mathrm{Cl}_{2}$ ) were as follows: step a, succinyl chloride, triethylamine (Et ${ }_{3} \mathrm{~N}$ ), and $\mathrm{N}, \mathrm{N}$-dimethyformamide (DMF) $0^{\circ} \mathrm{C}, 2$ hours, $80 \%$; step b, $t$-butyldiphenylsilyl chloride (TBDPSCl), $\mathrm{NH}_{4} \mathrm{NO}_{3}, \mathrm{DMF}, 8$ hours, $30 \%$; step c, trityl chloride ( $\mathrm{TrCl}$ ), $\mathrm{Bu}_{4} \mathrm{NClO}_{4}, 2,4,6$-collidine, $\mathrm{CHCl}_{3}, 61^{\circ} \mathrm{C}, 2$ hours, $86 \%$; step d, $\mathrm{NaBH}_{4}, \mathrm{LiCl}$, tetrahydrofuran and ethanol, 8 hours, 79\%; step e, FmocNH $\left(\mathrm{CH}_{2}\right)_{10} \mathrm{CO}_{2} \mathrm{H}$, dicyclohexylcarbodiimide (DCC), 1hydroxybenzotriazole (HOBt), 4-(dimethylamino)pyridine, 8 hours, $85 \%$; step $\mathrm{f}_{1} \mathrm{Et}_{2} \mathrm{NH}, 8$ hours, $73 \%$; step g, fumaric acid, DCC, $\mathrm{HOBt}, \mathrm{CHCl}_{3}, 8$ hours, $52 \%$; step h, 8 equivalents of isophthaloyl dichloride, 8 equivalents of $p$-xylylenediamine, $\mathrm{Et}_{3} \mathrm{~N}, \mathrm{CHCl}_{3}, 3$ hours, $30 \%$; step i, tetrabutylammonium fluoride (TBAF), 20 min then cool to $-10^{\circ} \mathrm{C}$ and add $2,4,6$-collidine, $t$-butyldimethylsilyl triflate (TBDMSOTf), $40 \mathrm{~min}$, overall $72 \%$; step j, hv $254 \mathrm{~nm}, 5 \mathrm{~min}, 50 \%$; step k, TBAF, 20 min then cool to $-78^{\circ} \mathrm{C}$ and add 2,4,6-collidine, TBDMSOTf, 1 hour, overall $61 \%$; step l, piperidine, 1 hour, $\sim 100 \%$; step $\mathrm{m}, \mathrm{Me}_{2} \mathrm{~S} \cdot \mathrm{BCl}_{3},-10^{\circ} \mathrm{C}, 10 \mathrm{~min}$, and then $\operatorname{TrCl}, \mathrm{Bu}_{4} \mathrm{NClO}_{4}, 2,4,6$-collidine, 16 hours, overall $74 \%$; step $\mathrm{n}, \mathrm{Me}_{2} \mathrm{~S} \cdot \mathrm{BCl}_{3},-10^{\circ} \mathrm{C}, 15 \mathrm{~min}$, then cool to $-78^{\circ} \mathrm{C}$ and add 2,4,6-collidine, TrOTf, 5 hours, overall $63 \%$; step o, TBAF, $20 \mathrm{~min}$, then cool to $-10^{\circ} \mathrm{C}$ and add $2,4,6$-collidine, TBDMSOTf, $40 \mathrm{~min}$, overall $76 \%$. The above refer to preparative yields of isolated compounds. Net clockwise circumrotation was also achieved in one pot through the successive addition of reagents to fum- $E-1$ in the following sequence ( ${ }^{1} \mathrm{H}$ NMR-determined percentage conversions are given in parentheses): step j, hv $254 \mathrm{~nm}, 5 \mathrm{~min}$ (50\% mal-Z-1 present); step k, 4 equivalents of TBAF, 20 min, cool to $-78^{\circ} \mathrm{C}$ and add 30 equivalents of TBDMSOTf and 30 equivalents of 2,4,6-collidine, $30 \mathrm{~min}$, then 60 equivalents of Wang resin, Dowex MR mixed bed ion-exchange resin and activated $4 \AA$ molecular sieves, decant ( $\sim 33 \%$ succ-Z-1 present); step l, 20 equivalents of piperidine, 1 hour, then Dowex MR mixed bed ion-exchange resin, decant ( $\sim 33 \%$ succ- $E-1$ present); step $m, 1$ equivalent of $\mathrm{Me}_{2} \mathrm{~S} \cdot \mathrm{BCl}_{3},-10^{\circ} \mathrm{C}, 5 \mathrm{~min}$, and then add 25 equivalents of 2,4,6-collidine and 20 equivalents of TrOTf, 1 hour ( $\sim 90 \%$ fum- $E-1$ present; $\sim 28 \%$ of the total molecules in the sample having undergone circumrotation). The chirality of 1 is present only for synthetic convenience. agents and byproducts, with only modest reductions in yields indicated by ${ }^{1} \mathrm{H}$ nuclear magnetic resonance (NMR) spectroscopy.

Unlike the previously reported (17) [3]catenane rotor, which relied on the dynamics of model compounds to determine stimuli-induced unidirectional behavior, the sense of rotation in $\mathbf{1}$ was demonstrated directly by isolation of samples of the [2]catenane after each synthetic step and the position of the small ring unambiguously determined by ${ }^{1} \mathrm{H}$ NMR (Figs. 4 and 5). Shielding effects reveal the position of the small macrocycle in each of the four catenane isomers. Comparison of the spectrum of fum-E-1 (Fig. 4A) with that of the macrocycle $E-2$ (Fig. 4B) shows a downfield shift of the $\mathrm{H}_{j}$ protons of the fumaramide station but not the succinamide group $\left(\mathrm{H}_{b}\right)$ in the catenane. In contrast, succ-E-1 (Fig. 4C) features a -1.3 parts per million difference in the $\mathrm{H}_{b}$ protons but little change in the signals of the fumaramide residue. The macrocycle is similarly located over the succinamide residue in succ-Z-1 (see, for example, the shifts of $\mathrm{H}_{b}$ in Fig. 5, B and C). Finally, although the small ring does not spend too much time actually over the poorly binding maleamide station in mal-Z-1 (as evidenced by the relatively small shift in $\mathrm{H}_{j}$ in Fig. 5A as compared to Fig. 5B), it is clearly trapped on the maleamide side of the silyl and trityl blocking groups because of the lack of shielding of the succinamide signals $\left(\mathrm{H}_{b}\right.$ are unchanged between Fig. 5A and Fig. 5B) and some significant shielding observed for $\mathrm{H}_{h}$.

Biological motors are too complex for the thermodynamic function of individual amino acid movements to be unravelled in detail. In contrast, the apparent simplicity of $\mathbf{1}$ and the minimalist nature of its design allow insight into the fundamental role that each part of the structure plays in the operation of the rotary machine.

The various chemical transformations perform two different functions: One pair (the linking/unlinking reactions, steps $\mathrm{k}$ and $\mathrm{m}$ or $\mathrm{n}$ and o in Fig. 3) modulates whether the small macrocycles can be exchanged between the two binding sites on the big ring (that is, allow the small macrocycle to become statistically balanced between the two binding sites according to a Boltzmann distribution); the second pair (balance-breaking reactions, steps $\mathrm{j}$ and 1 in Fig. 3) isomerize the olefin station (either $E \rightarrow Z$ or $Z \rightarrow E$ ), switching its binding affinity for the small macrocycle either on or off (37). By changing the relative binding affinities of the two stations in the large ring, each balance-breaking stimulus provides a driving force for redistribution of the small ring if it is able to move between the binding sites. In other words, the stations and blocking groups effectively disconnect the thermodynamic and kinetic components of detailed 
balance $(22,23)$; the balance-breaking reactions control the thermodynamics and impetus for net transport by biased Brownian motion, and the linking/unlinking reactions largely (38) control the relative kinetics and ability to exchange. Raising a kinetic barrier also "ratchets" transportation, allowing the statistical balance of the small ring to be subsequently broken without reversing the preceding net transportation sequence. Lowering a kinetic barrier allows "escapement" of a ratcheted quantity of rings in a particular direction.

To obtain $360^{\circ}$ rotation of the small ring about the large ring, the four sets of reactions must be applied in one of two sequences, each taking the following form: first a balancebreaking reaction; then a linking/unlinking step; then the second balance-breaking reaction; finally, the second linking/unlinking step. The direction of net rotation is determined solely by the way in which the balancebreaking and linking/unlinking steps are paired: an external input of information. The efficiency or yields of the reactions or the position of the ring at any stage (even if the machine makes a "mistake") are immaterial to the direction in which net motion occurs, as long as the reactions continue to be applied in the same sequence. Although reversing the sequence of the four steps changes the pairings and so rotates the small ring in the opposite direction, reversing the entire sequence of six chemical reactions does not, because linking/unlinking operations are not commutative.

We finish the circumrotation reaction sequence with the same molecule, fum-E-1, that we started with (although a $360^{\circ}$ rotation of one fragment has occurred), and the lightfueled balance-breaking reaction is reversed by a quantitative exoergic reaction, so no net energy is consumed to fuel the rotation. This is in one sense obvious, because the movement of the small ring takes place through Brownian motion and does not have to be powered; and in another somewhat surprising, because directional circumrotation of the small ring has occurred, which could be used to do external work - analogous to the hypothetical lifting of a flea by the SmoluchowskiFeynman ratchet and pawl (4). However, if the [2]catenane does do work against an opposing force, the energy required is taken from the balance-breaking reactions. If the balance-breaking chemical reactions do not actually fuel the net rotation, is it really necessary to carry them out? At first glance it seems that the small ring in a [2]catenane such as $\mathbf{3}$ might undergo directional circumrotation without them (Fig. 6).

Let us consider the situation in which $100 \%$ of the small rings are initially located on the succinamide station closest to the blocking groups: $100 \%$ succ 1-3. De-tritylation fol- lowed by re-tritylation generates 50\% succ2-3 (and 50\% remains as succl-3). Treatment of this mixture with a de-silylation/re-silylation sequence leads to a final product mixture, which again is $50 \%$ succ $1-3$ and $50 \%$ succ $2-3$.

However, half of the molecules that are now

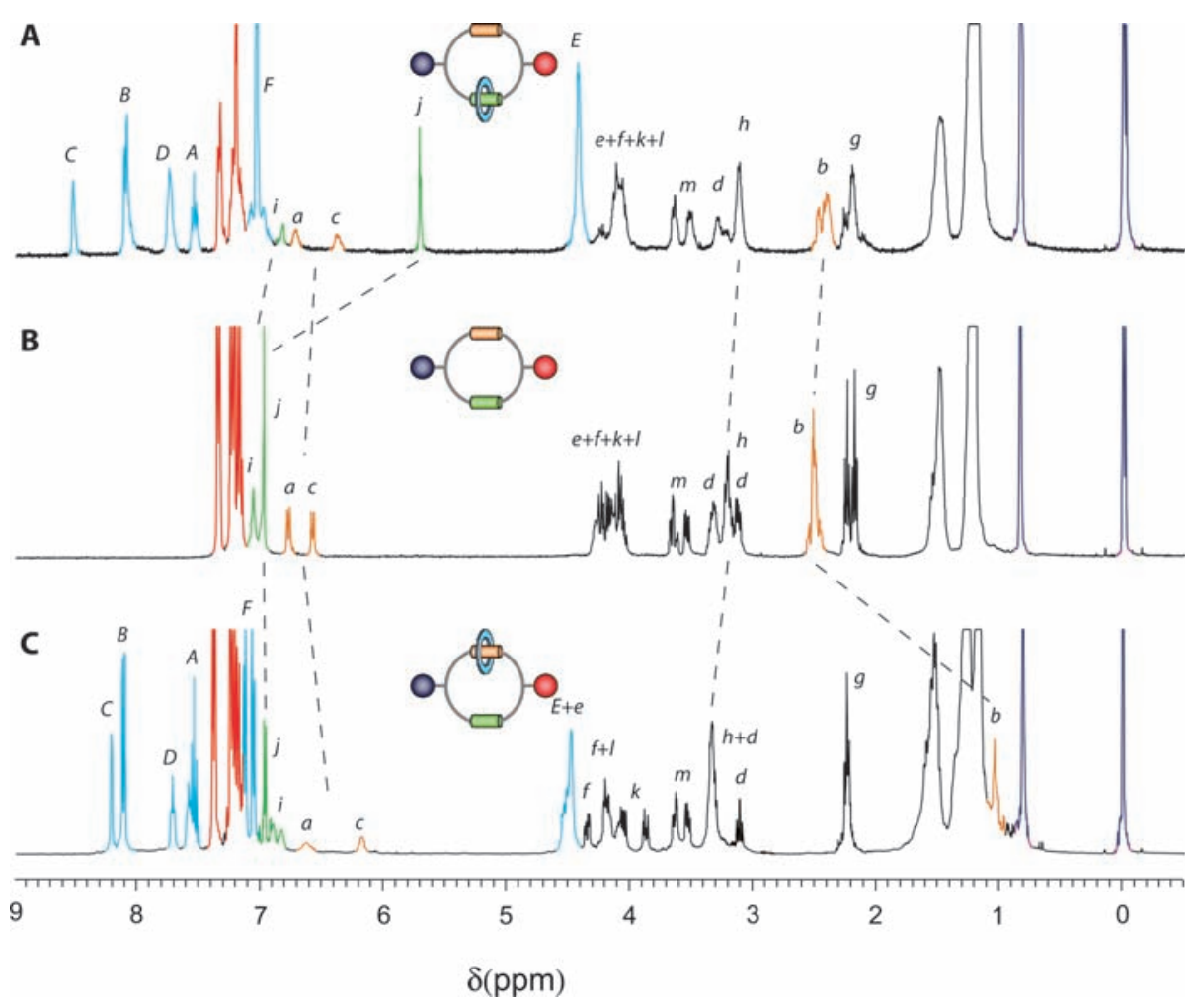

Fig. 4. ${ }^{1} \mathrm{H}$ NMR spectra $\left(400 \mathrm{MHz}, \mathrm{CDCl}_{3}\right.$, at $298 \mathrm{~K}$ ) of (A) [2]catenane fum- $E-1$, (B) macrocycle $E-2$, and (C) [2]catenane succ- $E-1$. The color coding and lettering correspond to those shown in Fig. 3.

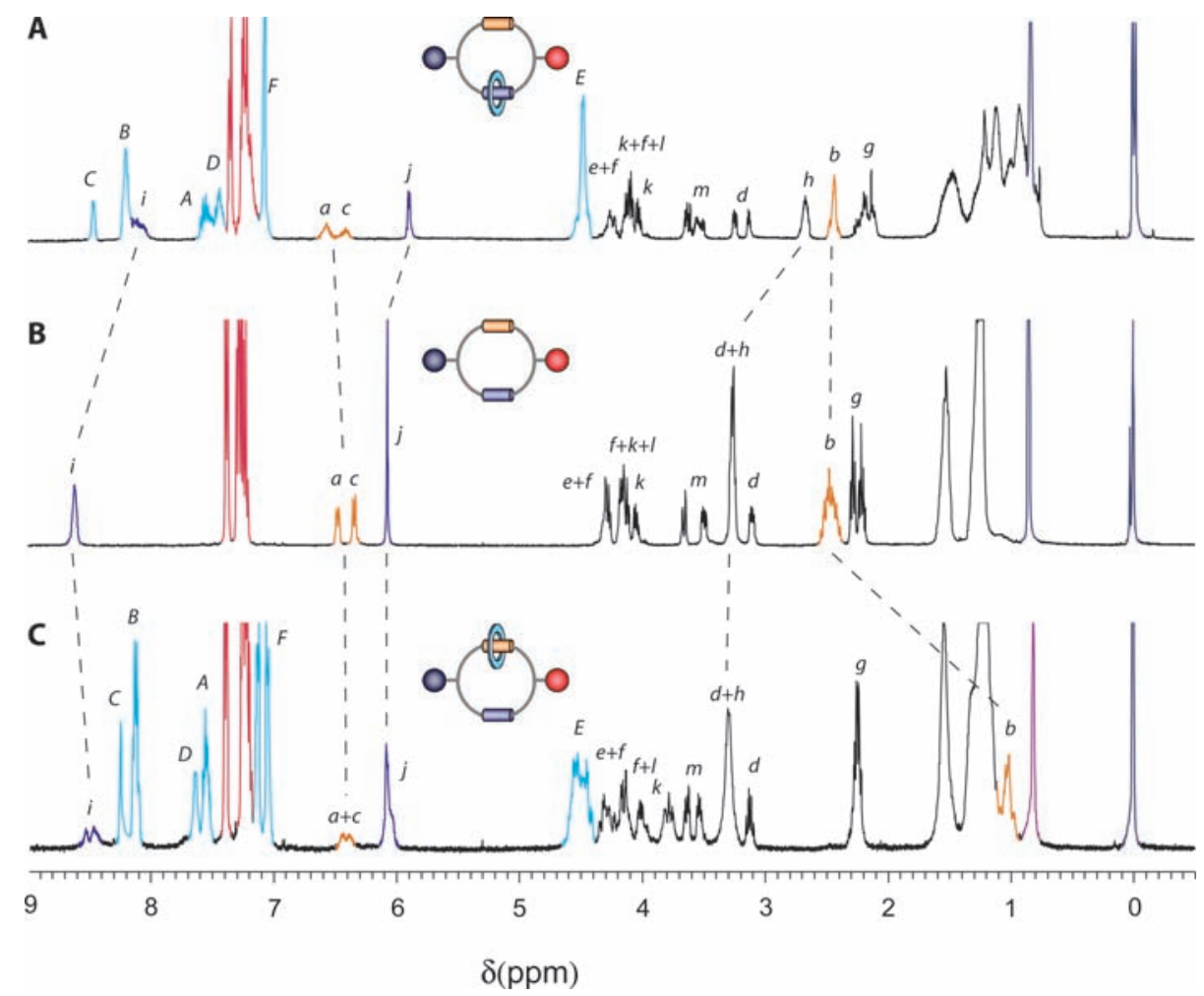

Fig. 5. ${ }^{1} \mathrm{H}$ NMR spectra $\left(400 \mathrm{MHz}, \mathrm{CDCl}_{3}\right.$, at $298 \mathrm{~K}$ ) of (A) [2]catenane mal-Z-1, (B) macrocycle Z2 , and (C) [2]catenane succ-Z-1. 


\section{R E P O R T S}

succ 1-3 (25\% of the total molecules) have undergone a $360^{\circ}$ clockwise circumrotation of the small ring, and all of the succ2-3 structures $(50 \%$ of the total molecules) have undergone a $180^{\circ}$ rotation of the small ring (half clockwise, half counterclockwise). The remaining $25 \%$ of total molecules have undergone no net positional change of the small ring, and so the average for the sample is a $90^{\circ}$ clockwise rotation without applying any chemical reactions to change the relative values of the two stations' energy minima. When we apply the sequence of reactions for a second (or any subsequent) time, however, although $75 \%$ of the molecules again individually undergo partial or complete revolution of the small ring, no net rotation occurs in either direction over a bulk sample. The $50 \%$ of molecules that start as succ 1-3 give a net $90^{\circ}$ clockwise rotation in response to the linking/unlinking sequence, and the $50 \%$ that start as succ $2-3$ rotate an equivalent amount in a counterclockwise sense. A statistically significant number of unidirectional rotation if the distribution of the small macrocycle between the binding sites is unbalanced at the start of the reaction sequence.

We can use some simple thermodynamic accountancy to calculate both the maximum amount of mechanical work that can be done by $\mathbf{1}$ and how much chemical energy has to be processed to cause directional motion of the against an opposing external force. The energy available to do work each time the small ring changes station equates to the macrocycle binding energy differences between the two sites (32). The light-fueled $E \rightarrow Z$ transformation allows up to $\Delta E_{\text {(small ring in succ-Z-1) }}$ $\Delta E_{\text {(small ring in mal-Z-1) }}$ to be performed during the subsequent linking step and the piperidinecatalyzed $Z \rightarrow E$ reaction permits a further $\Delta E_{\text {(small ring in fum-E-1) }}-\Delta E_{\text {(small ring in succ-E-1) }}$ catenane 3 molecules can only undergo net small ring even if no work needs to be done

(Fig. 3). Because $\Delta E_{\text {(small ring in succ-Z-1) }} \sim$ $\Delta E_{\text {(small ring in succ-E-1) }}$, the maximum total mechanical work that can be performed by circumrotation is $\Delta E_{\text {(small ring in fum-E-1) }}$ $\Delta E_{\text {(small ring in mal-Z-1) }}$; that is, the difference in small ring binding affinity between the fumaramide and maleamide stations. It is interesting to note that the binding affinity of the intermediate station is irrelevant to the amount of work that can be carried out by the molecular motor.

A quantitative thermodynamic requirement for unidirectional rotation of the small ring is provided by the difference between the free energies of the sequence of reactions $\mathrm{j}, \mathrm{k}, 1, \mathrm{~m}$ carried out on fum-E-1 and the same series of reactions applied to the macrocycle, $E-2$. The thermodynamics associated with the linking/unlinking steps cancel out (39). However, the $E \rightarrow Z$ isomerization step (step j) for the catenane is inherently more endothermic than the analogous reaction on the macrocycle because of the additional energy necessary to disrupt the hydrogen bond network of the small ring with the fumaramide station. The extra energy required to raise the potential energy of the transported particle (again, the difference in small ring binding energies of the fumaramide and maleamide stations) is returned to the thermal bath upon reisomerization to fumaramide and subsequent repositioning of the macrocycle. This requirement of extra energy to be processed by the system for the molecular machine to rotate directionally even when no work has to be done against an external force is once more reminiscent of the Smoluchowski-Feynman ratchet and pawl, which Feynman appreciated could be driven directionally by Brownian motion using heat flow between two thermal reservoirs; for example, by having the vanes attached to the shaft of the Feynman ratchet be hot and the wheel cold (40-42). We note that the amount of additional chemical energy that must be processed for directional rota-

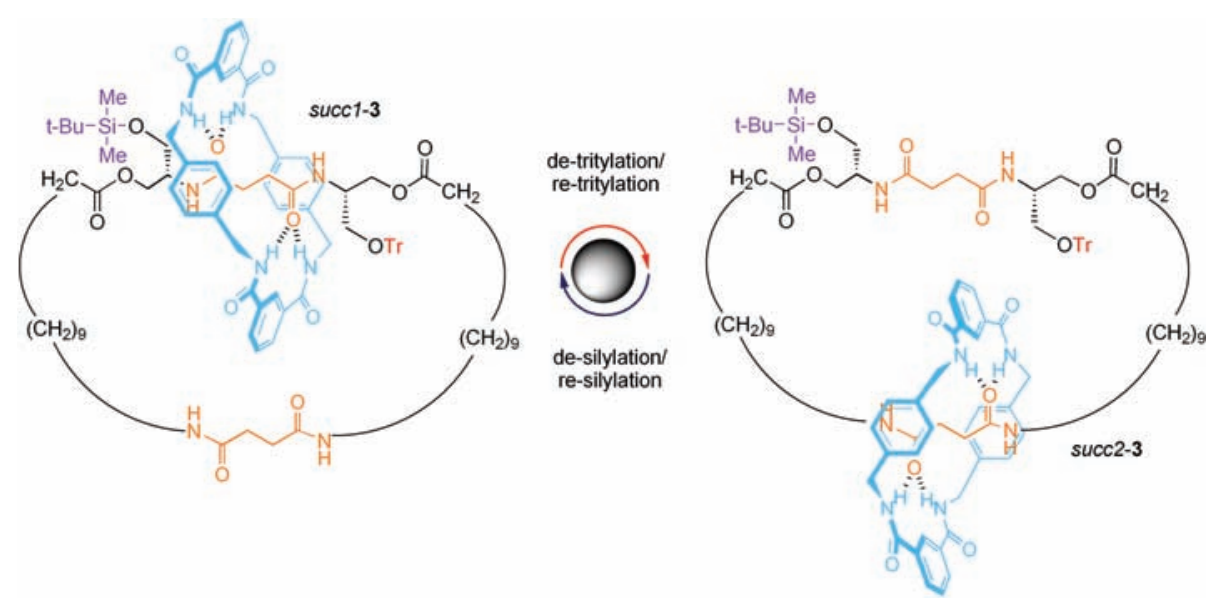

Fig. 6. Positional isomerism in a [2]catenane, 3, with stations of degenerate binding energies. tion to occur in the catenane is the sum of the energy differences that govern the Boltzmann distribution of the small ring between the binding sites at each stage; that is, the factor that determines the directional efficiency of the motor at constant temperature.

Mechanisms formulated from nonequilibrium statistical mechanics can be successfully used to design synthetic molecular motors such as 1. In turn, the analysis of this deceptively simple molecule, particularly the separation of the kinetic and thermodynamic requirements for detailed balance, provides experimental insight into how and why an energy input is essential for directional rotation of a submolecular fragment by Brownian motion. Even though no net energy is used to power the motion, there has to be a particular amount of energy conversion for net rotation to be directional over a statistically significant number of molecules; a requirement that is absent if the equivalent motion is nondirectional (or, indeed, for directional rotation within a single molecule where balance is inherently broken). The quantity of energy conversion required to induce directionality has an intrinsic lower limit, corresponding exactly (and somewhat beautifully) to both the energy difference that determines the directional efficiency of rotation and the maximum amount of work that the motor can theoretically perform in a single cycle; there can be no perpetually unidirectionally rotating molecular structure of the second kind through such a mechanism. The factors that determine the sense of rotation in $\mathbf{1}$, together with the requirement for finite energy conversion for directional rotation in circumstances when no work is done against an external force, illustrate how interplay between informational and thermodynamic laws governs directional Brownian rotation in molecular structures.

References and Notes

1. E. M. Purcell, Am. J. Phys. 45, 3 (1977).

2. M. Schliwa, Ed., Molecular Motors (Wiley-VCH, Weinheim, Germany, 2003).

3. P. D. Boyer, Angew. Chem. Int. Ed. 37, 2296 (1998).

4. R. P. Feynman, R. B. Leighton, M. Sands, The Feynman Lectures on Physics (Addison-Wesley, Reading, MA, 1963), vol. 1, chap. 46.

5. M. von Smoluchowski, Physik. Zeitschr. 13, 1069 (1912).

6. T. R. Kelly, I. Tellitu, J. P. Sestelo, Angew. Chem. Int Ed. Engl. 36, 1866 (1997).

7. A. P. Davis, Angew. Chem. Int. Ed. 37, 909 (1998).

8. V. Balzani, A. Credi, F. M. Raymo, J. F. Stoddart, Angew. Chem. Int. Ed. 39, 3349 (2000).

9. M. F. Hawthorne et al., Science 303, 1849 (2004).

10. C. P. Mandl, B. König, Angew. Chem. Int. Ed. 43, 1622 (2004).

11. T. R. Kelly, H. De Silva, R. A. Silva, Nature 401, 150 (1999).

12. T. R. Kelly, R. A. Silva, H. De Silva, S. Jasmin, Y. J. Zhao, J. Am. Chem. Soc. 122, 6935 (2000).

13. N. Koumura, R. W. J. Zijlstra, R. A. van Delden, N. Harada, B. L. Feringa, Nature 401, 152 (1999).

14. N. Koumura, E. M. Geertsema, M. B. van Gelder, A. Meetsma, B. L. Feringa, J. Am. Chem. Soc. 124, 5037 (2002). 
15. M. K. J. ter Wiel, R. A. van Delden, A. Meetsma, B. L. Feringa, J. Am. Chem. Soc. 125, 15076 (2003).

16. R. A. van Delden, M. K. J. ter Wiel, H. De Jong A. Meetsma, B. L. Feringa, Org. Biomol. Chem. 2, 1531 (2004).

17. D. A. Leigh, J. K. Y. Wong, F. Dehez, F. Zerbetto, Nature 424, 174 (2003).

18. P. Reimann, Phys. Rep. 361, 57 (2002).

19. J. M. R. Parrondo, L. Dinís, Contemp. Phys. 45, 147 (2004).

20. R. D. Astumian, Science 276, 917 (1997)

21. Randomizing influences other than Brownian motion can be used in ratchet mechanisms. For example, in quantum ratchets, quantum effects such as tunneling play this role.

22. The principle of detailed balance (23) tells us that no net task can be performed by the fluxional exchange of components at equilibrium, because transitions take place at the same rate in opposite directions. The structure of catenane 1 is remarkable in that it separates the kinetic (ability to exchange) and thermodynamic (impetus for net transport) requirements for detailed balance.

23. L. Onsager, Phys. Rev. 37, 405 (1931).

24. R. D. Astumian, P. Hänggi, Phys. Today 55, 33 (November 2002).

25. R. D. Astumian, I. Derényi, Eur. Biophys. J. 27, 474 (1998).

26. Special issue on "Ratchets and Brownian Motors:
Basics, Experiments and Applications," H. Linke, Ed., Appl. Phys. A 75, 167 (2002)

27. G. Oster, H. Y. Wang, Trends Cell Biol. 13, 114 (2003)

28. J. Rousselet, L. Salome, A. Ajdari, J. Prost, Nature $\mathbf{3 7 0}$, 446 (1994).

29. L. P. Faucheux, L. S. Bourdieu, P. D. Kaplan, A. J. Libchaber, Phys. Rev. Lett. 74, 1504 (1995)

30. J. S. Bader et al., Proc. Natl. Acad. Sci. U.S.A. 96 13165 (1999)

31. S. Matthias, F. Müller, Nature 424, 53 (2003).

32. A. Altieri et al., Angew. Chem. Int. Ed. 42, 2296 (2003).

33. The prefix indicates the position of the smaller macrocycle on the larger one.

34. Although catenane fum-E-1 was originally prepared as its $O$-t-butyldiphenylsilyl (TBDPS) derivative, the steric bulk caused low yields during the re-silylation protocol of step k, so it was replaced with the smaller TBDMS group for all the directional rotation studies.

35. Materials and methods are available as supporting material on Science Online.

36. The photoisomerization reaction gives a 50:50 E:Z mixture in the photostationary state. No accompanying photodegradation or decomposition was detected (37).

37. F. G. Gatti et al., Proc. Natl. Acad. Sci. U.S.A. 100, 10 (2003).

38. By raising or lowering the energy minima, the balance- breaking reactions intrinsically affect the kinetic barriers as well.

39. This is not quite as simple as the $\Delta G$ values of the forward and reverse covalent bond forming and breaking reactions canceling each other out. When unlinking takes place, the restriction in freedom of movement reduces the entropy of the small macrocycle in addition to the $\Delta G$ change associated with the chemical reaction; however, when linking occurs, the entropy increases by the same amount.

40. Certain aspects of Feynman's discussion of the ratchet and pawl as a motor have been shown to be flawed $(41,42)$.

41. J. M. R. Parrondo, P. Español, Am. J. Phys. 64, 1125 (1996).

42. M. O. Magnasco, G. Stolovitzky, J. Stat. Phys. 93, 615 (1998).

43. This work was supported by the Carnegie Trust (a scholarship to E.R.K.) and the European Union (a Marie Curie Fellowship to J.V.H.)

Supporting Online Material

www.sciencemag.org/cgi/content/full/306/5701/1532/ DC1

Materials and Methods

Scheme S1

11 August 2004; accepted 8 October 2004

\section{Three-Dimensional Hydrogen Microscopy in Diamond}

\author{
P. Reichart, ${ }^{1{ }^{*} \dagger}$ G. Datzmann, ${ }^{1}$ A. Hauptner, ${ }^{1}$ R. Hertenberger, ${ }^{2}$ \\ C. Wild, ${ }^{3}$ G. Dollinger ${ }^{1}$
}

\begin{abstract}
A microprobe of protons with an energy of 17 million electron volts is used to quantitatively image three-dimensional hydrogen distributions at a lateral resolution better than 1 micrometer with high sensitivity. Hydrogen images of a $<110>$-textured undoped polycrystalline diamond film show that most of the hydrogen is located at grain boundaries. The average amount of hydrogen atoms along the grain boundaries is $(8.1 \pm 1.5) \times 10^{14}$ per square centimeter, corresponding to about a third of a monolayer. The hydrogen content within the grain is below the experimental sensitivity of $1.4 \times 10^{16}$ atoms per cubic centimeter ( 0.08 atomic parts per million). The data prove a low hydrogen content within chemical vapor deposition-grown diamond and the importance of hydrogen at grain boundaries, for example, with respect to electronic properties of polycrystalline diamond.
\end{abstract}

Diamond is a promising material for various applications. Although its chemical and mechanical properties have led already to widespread applications, its potential for electronic or optical application remains limited because of imperfections (1). The recently demonstrated high carrier mobility in single-crystal chemical vapor-deposited diamond (2) opens

\footnotetext{
1Physik Department E12, Technische Universität (TU) München, 85748 Garching, Germany. ${ }^{2}$ Department für Physik, Ludwig-Maximilians-Universität (LMU) München, 85748 Garching, Germany. ${ }^{3}$ Fraunhofer Institut für Angewandte Festköperphysik, 79108 Freiburg, Germany.

*Present address: Microanalytical Research Centre School of Physics, University of Melbourne, Victoria, 3010, Australia.

†To whom correspondence should be addressed. E-mail: p.reichart@ph.unimelb.edu.au

Present address: Angewandte Physik und Messtechnik, Universität der Bundeswehr München, 85577 Neubiberg, Germany.
}

perspectives for electronic devices in hostile, high-voltage, or high-temperature environments (3); diamond optics (4); radiation sen-

However, the production and characterization of high-quality diamond, especially for electronic devices, is a great challenge. Chemical vapor deposition (CVD), for example, is a well-established method (7) to synthesize high-quality diamond layers and opens the possibility for hetero- and homoepitaxial growth of CVD diamond layers up to some mm thickness $(8,9)$. One main aim is to understand the origin of structural imperfections and impurities and to reduce or control these in order to improve the electronic and optical properties. The presence of hydrogen is known to influence these properties $(10,11)$. Depending on type and quality, average hydrogen concentrations were found from below the detection limits of sors (5); and particle detectors (6).
$10^{17}$ atoms $/ \mathrm{cm}^{3}$ up to about $10^{19}$ atoms $/ \mathrm{cm}^{3}$ in the bulk of polycrystalline diamond layers (12). Also, several hydrogen-related complexes were identified (13-15). However, there is insufficient information about the quantity and the spatial distribution of these, and perhaps as-yet-unidentified, types of hydrogen-related defects. It is even unknown whether most of the hydrogen being detected in diamond is concentrated at grain boundaries, inclusions, and other extended defects or whether hydrogen is distributed homogeneously throughout the bulk at defects of atomic dimensions. The decrease of the hydrogen content with increasing size of the crystallites inside polycrystalline CVD diamond layers, as well as infrared (15) or electron spin resonance studies (16), indirectly indicate that most of the hydrogen is situated at grain boundaries. Another explanation, however, is that there is reduced defect density within the grains with growing film thickness. Information about the absolute quantity of hydrogen inside the grain compared with

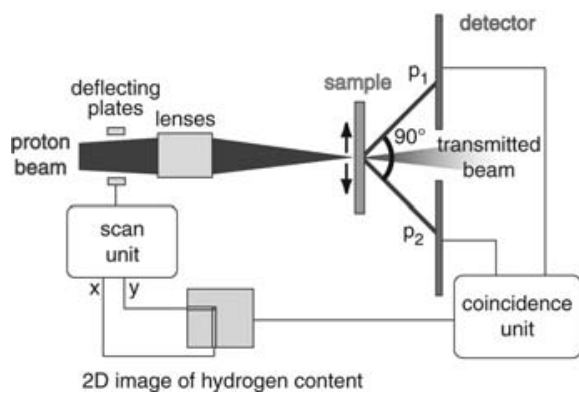

Fig. 1. Hydrogen analysis by pp scattering. The two protons emitted from a proton scattered at a hydrogen nuclei of the sample are detected in coincidence by a suitable detection system (25). 